\title{
TinkerType: a language for playing with formal systems
}

\author{
MICHAEL Y. LEVIN and BENJAMIN C. PIERCE \\ Department of Computer \& Information Science, University of Pennsylvania, \\ 200 South 33rd Street, Philadelphia, PA 19104, USA \\ (e-mail: \{milevin,bcpierce\}@cis.upenn.edu)
}

\begin{abstract}
TinkerType is a pragmatic framework for compact and modular description of formal systems (type systems, operational semantics, logics, etc.). A family of related systems is broken down into a set of clauses - individual inference rules - and a set of features controlling the inclusion of clauses in particular systems. Simple static checks are used to help maintain consistency of the generated systems. We present TinkerType and its implementation and describe its application to two substantial repositories of typed lambda-calculi. The first repository covers a broad range of typing features, including subtyping, polymorphism, type operators and kinding, computational effects, and dependent types. It describes both declarative and algorithmic aspects of the systems, and can be used with our tool, the TinkerType Assembler, to generate calculi either in the form of typeset collections of inference rules or as executable ML typecheckers. The second repository addresses a smaller collection of systems, and provides modularized proofs of basic safety properties.
\end{abstract}

\section{Introduction}

The quest for modular presentations of families of programming language features has a long history in the programming language community. Language designers since Landin $(1965 ; 1966)$ have understood how to view a multitude of high-level constructs through the unifying lens of the lambda-calculus. Further work has led to more structured approaches such as categorical semantics (Gunter, 1992; Mitchell, 1996; Jacobs, 1999), action semantics (Mosses, 1992), and monadic frameworks (Moggi, 1989). Using these tools, it is now possible to synthesize a variety of interpreters (Steele, 1994; Liang et al., 1995; Espinosa, 1995) and compilers (Liang \& Hudak, 1996; Harrison \& Kamin, 1998) from common blueprints or interchangeable building blocks.

For the type systems that accompany these languages, progress on unifying formalisms has been slower, though there have been some significant achievements in restricted domains, including Pure Type Systems (Berardi, 1988; Terlouw, 1989; Barendregt, 1992) and Sulzmann, Odersky and Wehr's generic treatment of type inference for systems of constrained types (1999); a related result outside the domain of programming languages is Basin, Matthews, and Viganò's modular presentation of modal logics in Isabelle (1995). In these proposals, the idea is to define a single 
"parameterized" system from which many particular systems can be obtained by instantiation. This method supports once-and-for-all proofs of properties like subject reduction and decidability that apply automatically to all instances. However, to give a single, parametric description of a collection of formal systems, we must first understand all the possible interactions among their features. If - as is common with type systems - some combinations of features are not well understood, then a less structured, more flexible approach is required.

The goal of the TinkerType project has been to develop a framework that facilitates compact and modular description of very diverse collections of formal systems, taking typed lambda-calculi as our driving example. We adopt a featurebased approach, breaking down a family of formal systems into a set of clauses annotated with a set of features chosen by the user to reflect the structure of the domain. In the domain of typed lambda-calculi, the clauses are individual inference rules, and features correspond to the presence of particular type constructors or structures such as subtyping or kinding in a given calculus. A clause may have multiple variants, each annotated with a set of relevant features (drawn from some set of atomic feature names) that control its inclusion in particular systems. A complete system is specified by a set of features.

Several things can go wrong in the process of maintaining a repository of features and clauses and extracting systems from it. A change in a clause may introduce inconsistencies with other variants of the same clause; a set of features identifying an extracted system may be nonsensical; the clauses of a system may turn out to be incompatible with each other. In our open-ended setting (the clauses themselves are uninterpreted strings as far as TinkerType is concerned), ensuring the "reasonableness" of generated systems is difficult in general. We have, however, identified several common sources of error in practice and introduced static consistency checks to help prevent them.

The contributions described in this paper are twofold. First, we present the TinkerType framework and describe its implementation. Secondly, we use it to classify a number of familiar typed lambda-calculi, including systems with subtyping, polymorphism, type operators and kinding, computational effects, and dependent types. Our first repository of typed lambda-calculi can be used to extract both inference-rule presentations of systems (as latex documents) and ML sources that can be compiled to produce running typecheckers and interpreters. Our second repository can be used to generate proofs of basic metatheoretic properties. (Strictly speaking, what we generate are proof scripts, whose correctness must be externally verified. This point is discussed in section 6.) These experiments represent substantial experience with using the TinkerType framework in practice.

The remainder of the paper proceeds as follows. In sections 2 and 3, we give precise definitions of the fundamental concepts underlying TinkerType: clauses, features and the process of composing systems and checking their consistency. Section 4 describes our implementation. Sections 5 and 6 present our two repositories for typed lambda-calculi. Sections 7 and 8 describe related work and discuss TinkerType in a broader context. In this paper, we mostly concentrate on applications of TinkerType to lambda-calculi and type systems, but its core mechanisms are actually 
quite general. In section 8 , we speculate on some potential applications in other domains.

The TinkerType implementation, user manual, and examples are available from http://www.cis.upenn.edu/ milevin/tinkertype.

\section{Assembling systems from features and clauses}

A formal system can be described as a set of judgements, each consisting of a set of clauses. The simply typed lambda-calculus $(\lambda \rightarrow)$, for example, is a formal system with two judgements: typing and evaluation. The typing judgement contains clauses like

$$
\frac{\Gamma \vdash \mathrm{t}_{1}: \mathrm{T}_{2} \rightarrow \mathrm{T}_{1} \quad \Gamma \vdash \mathrm{t}_{2}: \mathrm{T}_{2}}{\Gamma \vdash \mathrm{t}_{1} \mathrm{t}_{2}: \mathrm{T}_{1}}
$$

T-APP

while the evaluation relation has clauses like the beta-reduction rule. ${ }^{1}$

This is obviously a rather syntactic view of formal systems. More abstractly, we might say that the simply typed lambda-calculus is a pair of sets of derivation trees: one set of trees with conclusions like $\Gamma \vdash t: \mathrm{T}$ and one with conclusions like $t \rightarrow \beta t^{\prime}$. More abstractly yet, we might view $\lambda^{\rightarrow}$ as a pair of relations obtained from these sets of trees. Or again, $\lambda \rightarrow$ can be represented by a pair of functions in, say, ML. Since we are interested in all of these views, we avoid committing to a particular one by taking clauses as primary and dealing with them as uninterpreted atoms in our formalism.

A given clause may appear in many different systems. For example, both pure $\lambda \rightarrow$ and $\lambda \rightarrow$ with booleans contain the application rule shown above. On the other hand, in other systems, the same clause may take different forms. In (an algorithmic presentation of) $\lambda \rightarrow$ with subtyping, the application rule refines the rule above by adding an extra subtyping premise:

$$
\frac{\Gamma \vdash \mathrm{t}_{1}: \mathrm{T}_{2} \rightarrow \mathrm{T}_{1} \quad \Gamma \vdash \mathrm{t}_{2}: \mathrm{U} \quad \mathrm{U}<: \mathrm{T}_{2}}{\Gamma \vdash \mathrm{t}_{1} \mathrm{t}_{2}: \mathrm{T}_{1}}
$$

Another specialization of the rule is necessary for systems with assignment and store. The typing judgement of such systems involves an assignment of types to store locations $\Sigma$.

$$
\frac{\Gamma ; \Sigma \vdash \mathrm{t}_{1}: \mathrm{T}_{2} \rightarrow \mathrm{T}_{1} \quad \Gamma ; \Sigma \vdash \mathrm{t}_{2}: \mathrm{T}_{2}}{\Gamma ; \Sigma \vdash \mathrm{t}_{1} \mathrm{t}_{2}: \mathrm{T}_{1}}
$$

We formalize the relation between different versions of inference rules and properties of the system by annotating each rule with a set of features:

\footnotetext{
${ }^{1}$ Strictly speaking, there are also "judgements" defining the syntax of types, terms, and contexts. For example, the term syntax judgement contains clauses like "if $T_{1}$ and $T_{2}$ are types, then so is $T_{1} \rightarrow T_{2}$." Our discussion elides these syntactic judgements, for brevity.
} 


$$
\begin{array}{cr}
\frac{\Gamma \vdash \mathrm{t}_{1}: \mathrm{T}_{2} \rightarrow \mathrm{T}_{1} \quad \Gamma \vdash \mathrm{t}_{2}: \mathrm{T}_{2}}{\Gamma \vdash \mathrm{t}_{1} \mathrm{t}_{2}: \mathrm{T}_{1}} & \text { T-APP [arrow] } \\
\frac{\Gamma \vdash \mathrm{t}_{1}: \mathrm{T}_{2} \rightarrow \mathrm{T}_{1} \quad \Gamma \vdash \mathrm{t}_{2}: \mathrm{U} \quad \mathrm{U}<: \mathrm{T}_{2}}{\Gamma \vdash \mathrm{t}_{1} \mathrm{t}_{2}: \mathrm{T}_{1}} & \text { T-APP [arrow, sub] } \\
\frac{\Gamma ; \Sigma \vdash \mathrm{t}_{1}: \mathrm{T}_{2} \rightarrow \mathrm{T}_{1} \quad \Gamma ; \Sigma \vdash \mathrm{t}_{2}: \mathrm{T}_{2}}{\Gamma ; \Sigma \vdash \mathrm{t}_{1} \quad \mathrm{t}_{2}: \mathrm{T}_{1}} & \text { T-APP [arrow, store] }
\end{array}
$$

The choice of features is determined by the set of systems we intend to describe. The arrow feature is present in any system with function types; sub characterizes systems with subtyping, and store indicates systems with locations and side effects.

Often features are related to one another. A system with higher order functions, for instance, necessarily has term variables because of lambda abstraction. Similarly, the Top type and its associated inference rules are only sensible in systems with subtyping. To account for facts like this, it is convenient to introduce the notion of dependencies between features. The arrow feature depends upon tmvar (written as a propositional formula arrow $\Rightarrow$ tmvar); top depends on $s u b$ (written top $\Rightarrow s u b$ ). Both of these dependencies are of the form one feature implies another. Sometimes, it is useful for a combination of multiple features to trigger a dependency. Consider, for example, a typing rule for conditional expressions (present in systems identified by the feature bool):

$$
\frac{\Gamma \vdash t_{1}: \text { Bool } \quad \Gamma \vdash \mathrm{t}_{2}: \mathrm{T} \quad \Gamma \vdash \mathrm{t}_{3}: \mathrm{T}}{\Gamma \vdash \text { if } \mathrm{t}_{1} \text { then } \mathrm{t}_{2} \text { else } \mathrm{t}_{3}: \mathrm{T}}
$$

In the presense of subtyping, a more flexible typing rule is possible. Instead of requiring the types of both conditional branches to be equal, it is safe to allow them to be different and assign their least common supertype, or join, to the type of the whole if expression:

$$
\begin{gathered}
\Gamma \vdash \mathrm{t}_{1}: \mathrm{T}_{1} \quad \mathrm{~T}_{1}<\text { : Bool } \quad \Gamma \vdash \mathrm{t}_{2}: \mathrm{T}_{2} \\
\Gamma \vdash \mathrm{t}_{3}: \mathrm{T}_{3} \quad \mathrm{~T}=\mathrm{T}_{2} \vee_{\mathrm{T}_{3}} \\
\Gamma \vdash \text { if } \mathrm{t}_{1} \text { then } \mathrm{t}_{2} \text { else } \mathrm{t}_{3}: \mathrm{T}
\end{gathered}
$$

A system with this rule must provide a function for calculating joins. Let us associate the inference rules of this function with the feature calcjoin. Then, we can formalize our discussion by the dependency bool $\wedge$ sub $\Rightarrow$ calcjoin.

Given a repository of inference rules as above, one can specify a system by a set of features. Consider $\lambda^{\rightarrow}$ with booleans and Top: [arrow, bool,top]. Let us determine the inference rules composing this system. The rule T-App [arrow] is relevant since it is tagged with a feature appearing in the system specification. The rule T-ApP $[$ arrow, sub] is also relevant; even though sub does not appear in the specification directly, it is implied by top. Clearly, the system should include the more specific latter rule. Similarly, this system should contain T-IF [bool,sub] as well as the applicable subtyping and join rules not shown here.

Attempting a similar exercise for the system [arrow, sub, store] will hit a snag 
since both T-APP [arrow, sub] and T-ApP [arrow, store] are relevant but neither one is more specific than the other. To resolve this conflict, we must define a refined rule for this specific set of features:

$$
\frac{\Gamma ; \Sigma \vdash \mathrm{t}_{1}: \mathrm{T}_{2} \rightarrow \mathrm{T}_{1} \quad \Gamma ; \Sigma \vdash \mathrm{t}_{2}: \mathrm{U} \quad \mathrm{U}<: \mathrm{T}_{2}}{\Gamma ; \Sigma \vdash \mathrm{t}_{1} \mathrm{t}_{2}: \mathrm{T}_{1}} \quad \text { T-APP [arrow, sub, store] }
$$

We can formalize the above intuitions as follows. First, fix a set Names of clause names and a set Cnt of clause contents. (Both of these sets are uninterpreted here. In the implementation, they are strings.) A repository is a tuple $\langle$ Fts, Dep, Cls $\rangle$, where

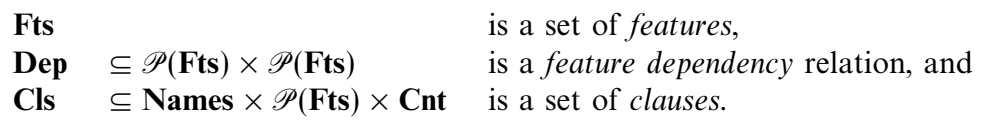

Given a set of features $F$, we define $\operatorname{closure}(F)$ to be the least superset $F^{\prime}$ of $F$ that is closed under the dependency relation, i.e. such that if $F_{1} \subseteq F^{\prime}$ and $\left(F_{1}, F_{2}\right) \in$ Dep, then $F_{2} \subseteq F^{\prime}$. We say that a set of features $F_{1}$ dominates another set $F_{2}$ if closure $\left(F_{2}\right) \subseteq \operatorname{closure}\left(F_{1}\right)$.

A clause $c l$ is a triple $\langle n, F, c\rangle$, where $n \in$ Names is a label identifying the clause, $F \subseteq$ Fts is a set of features that governs inclusion of the clause in particular systems, and $c \in \mathbf{C n t}$ is the actual content of the clause. We say that $c l$ is relevant to the set of features $F$. Finally, we say that a clause $c l_{1}=\left\langle n_{1}, F_{1}, c_{1}\right\rangle$ is more specific than $c l_{2}=\left\langle n_{2}, F_{2}, c_{2}\right\rangle$ if $n_{1}=n_{2}$ and $F_{1}$ dominates $F_{2}$. For example, T-IF [bool, sub] is more specific than T-IF [bool].

Now we have the tools to specify how a system is assembled, given a set of features $F$. First, we extract from the repository all the clauses whose sets of features are dominated by $F$. Then we partition these clauses into sets of clauses with identical labels. We verify that each partition has the most specific clause. We select the most specific clause from each partition. The contents of these clauses form the system.

\section{Consistency checking}

The basic framework presented in the previous section is very flexible, but it needs a little more structure before we can use it to develop large repositories. In this section, we introduce some simple static consistency checks that help ensure both coherence of the repository and consistency of generated systems. While these checks provide no absolute guarantees of correctness, we have found them to be extremely useful in practice.

To make the checks precise, we begin by adding some elements to the definitions of the previous section. A repository is now a tuple $\langle$ Fts, Dep, Cls, Rfn, Con, Csig $\rangle$, where

$\begin{array}{ll}\text { Fts, Dep, Cls } & \text { are as before, } \\ \mathbf{R f n} \subseteq \mathbf{C n t} \times \mathbf{C n t} & \begin{array}{l}\text { is a transitive refinement relation on clause contents, } \\ \text { is a set of feature constraint formulas (propositional formulas over }\end{array} \\ \mathbf{C s i g} & \begin{array}{l}\text { Fts), and } \\ \text { is a clause signature relation. }\end{array}\end{array}$

The new elements are discussed in the subsections that follow. 


\subsection{Clause refinement}

The refinement relation $\mathbf{R} \mathbf{f n}$ verifies that the dominance relation between clauses is reflected in their contents. For example, in the previous section we saw two variants of the application clause, T-ApP [arrow] and a more specific T-APP [arrow, sub]. It is natural to expect that the contents of these clauses should be similar, indeed that the contents of the latter clause should be "more specific" in the sense that it performs the same function but takes into account the presence of subtyping. If this were not the case, it would almost surely indicate that some confusion has happened in the repository (an inconsistent update of a clause content, an accidental name clash between clauses, incorrect feature dependency, mistaken feature annotation, etc.). To prevent such inconsistencies, we demand that, whenever a clause $\left\langle n, F_{1}, c_{1}\right\rangle$ is more specific than a clause $\left\langle n, F_{2}, c_{2}\right\rangle$, we also have $c_{1} \mathbf{R} \mathbf{f n} c_{2}$.

We need to take $\mathbf{R f n}$ as a part of the repository (i.e. we must assume that it is provided externally, not calculated by the framework) because the clause contents themselves are uninterpreted by the framework. (In our tool, the refinement relation is generated by user annotations explicitly marking the parts of clauses that are "new" with respect to previous versions, cf. section 4.) Forcing the user to think explicitly about the refinement relation between different versions of a clause introduces a useful cross-check between the activities of deriving new clauses from simpler variants and annotating them with features. Although this check works completely at the level of strings, it is surprisingly effective in catching "version control" errors during maintenance of large rule repositories: if we change a variable name, for example, in one variant of a clause but forget to change it in the other variants, the tool complains and points us to the ones we missed.

\subsection{Feature consistency}

In some families of formal systems, there are combinations of features that do not make sense. For example, it has been shown (Ghelli, 1990) that the subtype relation of the "full" variant of System $\mathrm{F}_{<:}$is not closed under joins. Other systems, like $\lambda \rightarrow$ with booleans and subtyping, rely on the existence of joins (signaled by the calcjoin feature) to calculate minimal types. Thus, a system like [ffsub, bool], where ffsub is the feature selecting the full variant of $\mathrm{F}_{<:}$, will be defective (in particular, the typing algorithm will be incomplete with respect to a declarative presentation of the system).

To prevent the extraction of such systems, we include in the repository a set Con of feature constraint formulas - propositional formulas over the set of features and say that a system identified by features $F$ is consistent if $\operatorname{closure}(F)$ satisfies every formula in Con. (Note, that feature dependencies are a special kind of constraints guaranteed to be satisfied by the definition of closure.) A constraint that outlaws the above system can be written ffsub $\Rightarrow \neg$ calcjoin.

\subsection{Judgement signatures}

Our last form of consistency checking is more speculative (and as yet unimplemented). From our experience using TinkerType, we feel that something of this kind 
is needed, but we are less confident about the details of the design: it seems difficult to strike the right balance between helping and getting in the way.

A given judgement may have different "shapes" when it appears in different formal systems. For example, in the simply typed lambda-calculus, the subtyping judgement is a two-place relation on types, $\mathrm{S}<: \mathrm{T}$. In $\mathrm{F}_{<\text {: }}$, it is a three-place relation between contexts and pairs of types, $\Gamma \vdash \mathrm{S}<$ : T. To track these variations in shape and prevent the accidental mixing of rules of different shapes, we can introduce a notion of judgement signatures. For example, in a type system with kinds, the signature of the typing judgement would be:

$$
\text { Typing }(\Gamma, \mathrm{t}, \mathrm{T}) \times \operatorname{Kinding}(\Gamma, \mathrm{T}, \mathrm{K}) \rightarrow \text { Typing }(\Gamma, \mathrm{t}, \mathrm{T})
$$

That is, typing in this system is a three-place relation on contexts, terms, and types, and it depends both on itself and on the kinding relation (i.e. both typing and kinding assertions may occur as major premises of typing rules). Formally, adding judgement signatures involves extending the definition of a repository with new sets Syn of syntactic categories and $\mathbf{J d g}$ of judgement names. A statement signature like Typing $(\Gamma, \mathrm{t}, \mathrm{T})$ consists of a judgement name (Typing) and a sequence of syntactic categories. A judgement signature has the form $S_{1} \times \cdots S_{n} \rightarrow S$, where each $S_{i}$ and $S$ are statement signatures.

Judgement signatures enable a consistency check that prevents clauses intended for different versions of a judgement from ending up in the same system. This is accomplished by checking, when assembling a system, that all the clauses we have selected to define a given judgement have exactly the same signature. This check alerts the user when a clause that should have been overridden to take a new feature into account is "improperly inherited" verbatim from a simpler system. For example, it will prevent the inclusion of the clause

$$
\frac{\Gamma, \mathrm{x}: \mathrm{T}_{2} \vdash \mathrm{t}_{1}: \mathrm{T}_{1}}{\Gamma \vdash \lambda \mathrm{x}: \mathrm{T}_{2} \cdot \mathrm{t}_{1}: \mathrm{T}_{2} \rightarrow \mathrm{T}_{1}}
$$

T-LAM $[$ arrow $]$

in a system with a kinding relation, because its signature is Typing $(\Gamma, \mathrm{t}, \mathrm{T}) \rightarrow$ Typing $(\Gamma, \mathrm{t}, \mathrm{T})$, rather than Typing $(\Gamma, \mathrm{t}, \mathrm{T}) \times \operatorname{Kinding}(\Gamma, \mathrm{T}, \mathrm{K}) \rightarrow$ Typing $(\Gamma, \mathrm{t}, \mathrm{T})$. This check should help prevent the generation of unsound or nonsensical systems - for example, when the user forgets to override the T-LAM clause with its kind-checking variant.

Our implementation does not support judgement signature checking yet: some substantial design issues remain to be addressed before the idea can be tried out in practice. In particular, since clause contents are uninterpreted (e.g. just strings), TinkerType cannot infer judgement signatures automatically; like the refinement relation on clauses, the relation mapping clauses to signatures must be specified by the user as part of the repository. Accomplishing this smoothly, without imposing an undue burden on the user, requires a mechanism for indicating judgement signatures for large collections of rules at once. Also, a straightforward realization of the consistency check prevents some legitimate uses of inheritance. Consider again the two type systems with and without kinding. Even though their typing 
judgements have different signatures, the application clause T-APP is identical in both systems (unlike T-LAM). We believe that silent inheritance in cases like this should be prohibited - the user should be forced to look at the simple clause and verify that it will actually work unchanged in a system with kinding, but we need to make it easy for the user to record the fact that this check has indeed been performed. In the meantime, the implementation provides a simpler consistency check that detects some of the same problems.

\section{The TinkerType assembler}

Based on the above ideas, we have designed a small language for describing repositories and implemented a tool that assembles systems and checks their consistency.

We use arbitrary strings for the contents of clauses. In our repositories of typed lambda-calculi, some of these strings are bits of ML code; others are bits of TeX source; others are bits of proofs. For example, here is the ML clause for typechecking conditional expressions:

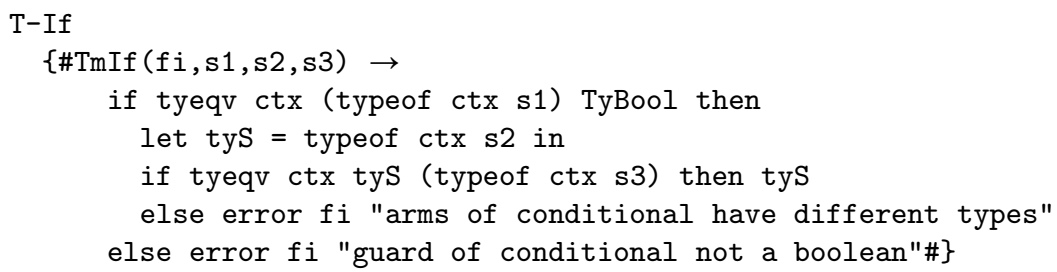

T-If is the name of the clause, and the content appears between the brackets $\{\#$ and \#\}. This clause forms part of the definition of the typeof function in a generated typechecker. (It can be paraphrased as follows: In the case where we are typechecking a TmIf abstract syntax node, we first check whether the type of the guard s1 (in the current context ctx) is equivalent to TyBool. If so, we calculate the type of the then part $s 2$ and call this type tys. We calculate the type of the else part s3 and check that it is equivalent to tys. If it is, then tys is the type of the TmIf node. If either test fails, we generate an appropriate error.) Individual clauses are not annotated with their relevant features. Instead, we introduce a coarsergrained structuring mechanism called a component, which gives a single annotation for several clauses relevant to the same set of features. Besides reducing clutter, components are useful units of grouping in the repositories.

Within a component, clauses are further grouped into nested sections. At the top level, we have one section for all the TeX rules and another for ML code. (We could also have decided to intermix TeX and ML clauses in the same sections, so that clauses implementing similar functionality would be adjacent in the repository sources; the choice is purely a matter of taste.) Within the ML section, there are subsections for abstract syntax, for lexing and parsing, for printing, and for core typechecking functions. The latter contains subsections for individual functions (typing, subtyping, kinding, etc.), and they, in turn, contain the actual clauses. The 
following fragment is a part of the [bool,typing] component:

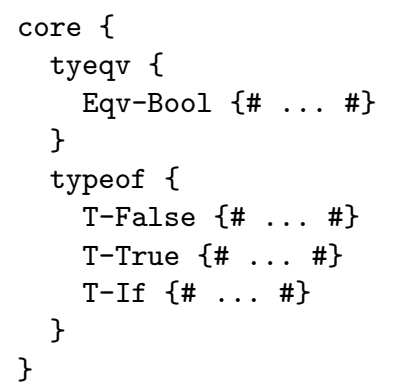

Each section may define special clauses called header, footer, and separator. For example, the typeof section contains the following header and separator:

header $\quad$ \#let rec typeof ctx $t=$ match $t$ with\#\}

separator $\{\# \mid \#\}$

(In the actual repositories, these definitions also contain information about linebreaking and indentation.) When the tool prints a section, it outputs the header, the section's subitems separated by the separator, and then the footer. For example, the generated typeof function looks like this:

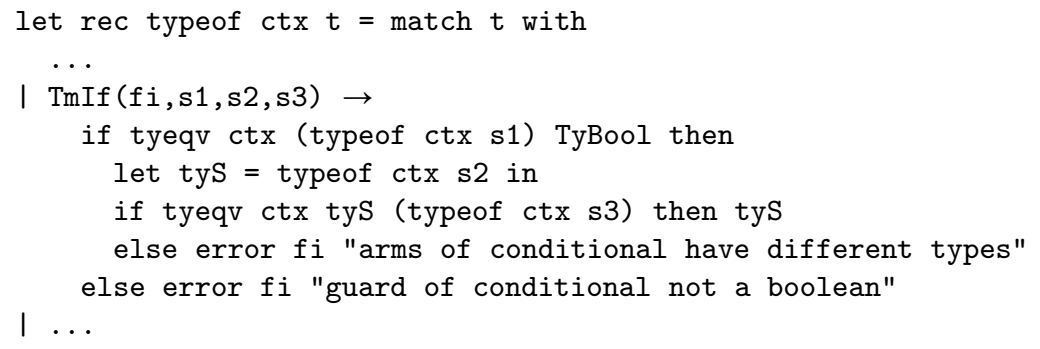

The ability to associate sections with headers, footers, and separators is the main practical motivation for the section mechanism (aside from this, we could simulate most of the uses of sections by adding auxiliary features). As the previous example shows, it is essential for composing ML function definitions from clauses of a pattern match. Outer sections, like the ones grouping all TeX rules and all ML code, are introduced mostly for readablility, and their layout is up to the taste of the component writer.

The refinement relation is generated from user annotations in clause contents. To indicate that one version of a clause refines another, we enclose the new or changed parts of the refined clause in [ [ and ] ] brackets. The assembler notes that the latter refines the former if the unbracketed segments of the refined clause appear verbatim in the original. For example, the variant of $\mathrm{T}-\mathrm{If}$ for systems with subtyping is annotated like this:

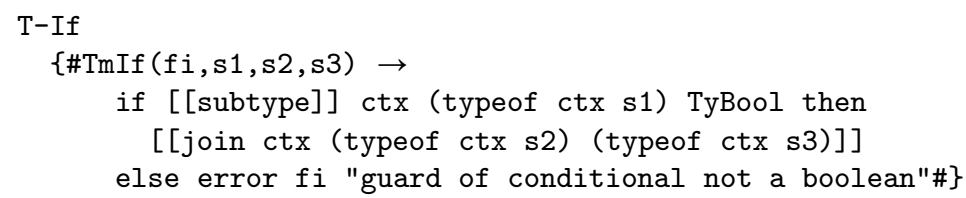


To build a system, we specify a set of features plus a file or directory, called a template, containing a skeleton for the generated system. For ML systems, the template is a directory containing a makefile, boilerplate for lexing and parsing, a top-level command loop, and skeleton modules (containing holes marked with the names of sections to be inserted at each point) for the major components of the typechecker.

The TinkerType implementation also includes fairly sophisticated facilities for prettyprinting, controlling the ordering of clauses and sections, automatic highlighting of differences between a generated system and its ancestors, macro substitution, debugging support for generated systems, and analysis of feature conflicts and rule inconsistencies. Details can be found in the user manual (available through http:// www. cis . upenn. edu/ milevin/tinkertype).

\section{The Next 700 type systems}

We have carried out two substantial experiments with using TinkerType. In the first, the goal was to encode a very broad range of typing features, including subtyping, polymorphism, type operators and kinding, computational effects, and dependent types, and to develop both printable TeX presentations and executable ML typecheckers for the systems in parallel. In the second, we addressed a smaller collection of features, concentrating instead on modularizing the proofs of their basic metatheory. This section describes the first experiment; the second is discussed in section 6 .

In the following subsections, we introduce several groups of related features and show how to combine them to obtain several familiar type systems, including the systems of Barendregt's lambda cube and various calculi with subtyping. We concentrate on the algorithmic variants of the systems (i.e. the running ML systems, rather than their more abstract presentations in TeX), since they involve a more interesting "feature skeleton" than their declarative counterparts. Each of the following subsections shows one "slice" through our repository, introducing several related features and discussing their use.

\subsection{Features for variables and binders}

Some of the intricacies in the repository arise from the need to deal carefully with (term and type) variables and substitution. In particular, we would like to make each generated system as simple as possible, avoiding generating unnecessary functionality like type-substitution operations in systems whose types do not contain variables. We must therefore take into account whether type and term variables are present in each system, whether type and term variables can appear in terms and types (respectively), and which substitution operations (terms in terms, types in terms, types in types, etc.) are needed. (Remember that we are generating running ML typecheckers here; the standard "We assume the usual conventions about alpha-conversion and capture-avoiding substitution..." does not suffice! We could, of course, alternatively adopt a "higher-order abstract syntax" treatment of binders and substitution, as in 

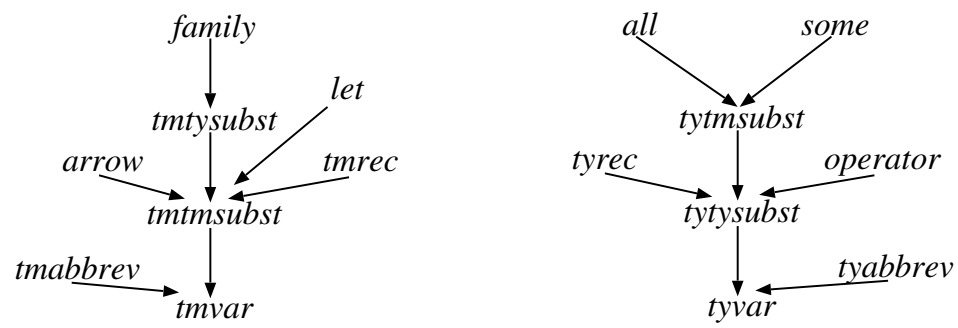

Fig. 1. Variable and binding features.

LF (Harper et al., 1992) and some concrete compiler implementations (Pfenning \& Elliott, 1988). Our choice of an explicit treatment of names is a matter of taste, not principle: TinkerType could be used equally well to formulate the HOAS variants of our systems.)

Figure 1 shows the hierarchy of features related to variables and binders. The two "trunks" in the diagram control the core functionality of variables and substitution. We use the features tmtmsubst, tmtysubst, tytmsubst, and tytysubst to control the generation of functions for substitution of terms inside terms, terms inside types, types inside terms, and types inside types respectively. The "leaves" in the hierarchies represent specific binding operators whose definitions make use of various kinds of substitution. The features arrow and family in the term-variable hierarchy (on the left) stand for abstraction of terms over terms (ordinary lambda-abstraction) and types over terms (families of types indexed by terms). They correspond to the operator and all features in the type variable hierarchy, which characterize abstraction of types over types (type operators) and terms over types (polymorphic functions), respectively. The feature let stands for local definitions, some for existential types, tmrec and tyrec for recursive terms and iso-recursive types, variant for variants and a case construct, and tmabbrev and tyabbrev for top-level abbreviations of terms and types.

Note that "technical features" like tytmsubst do not need to be mentioned in userlevel descriptions of systems, since mentioning a higher-level feature like all will cause it to be included automatically. Also, note that tytmsubst implies tytysubst, since substituting a type into a term might involve substituting through a type embedded in the term, and similarly for tmtysubst and tmtmsubst.

The variable related features contain the cornerstones for the systems of Barendregt's lambda cube: arrow, all, operator, and family. We will return to the cube later on.

\subsection{Simple features}

Another way to classify features is based on the kinds of judgements they support. The feature typing enables the typing judgement. A large number of systems can be built based on this judgement. We call such systems simple, and figure 2 shows the hierarchy of features used to build them.

The simple feature hierarchy includes almost all of the variable operation hierarchy 


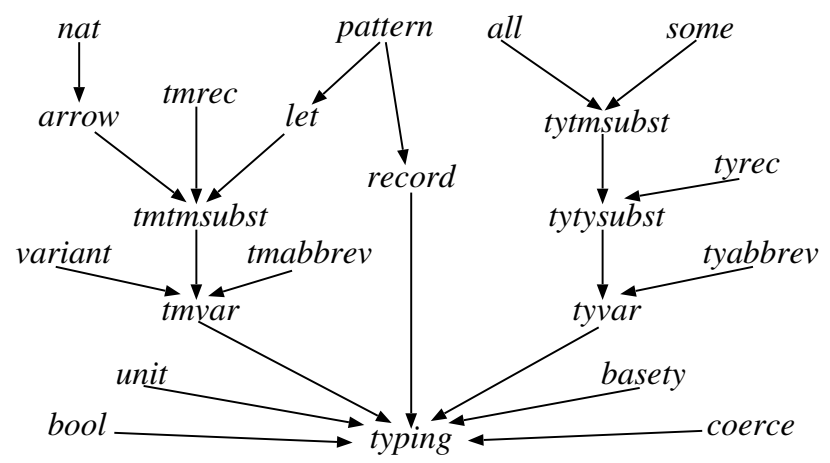

Fig. 2. Simple features

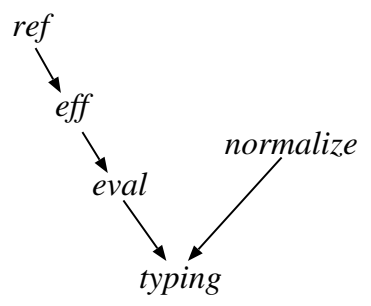

Fig. 3. Evaluation and normalization features.

and defines several new "content" features. The feature nat represents natural numbers and the operation of iteration on them. Because one argument of such iterations is a higher-order function, nat implies arrow. The feature pattern combines the capabilities of local definition (let) and record projection (record) to enable pattern matching syntax for record values. The features bool and unit represent booleans and a unit type; basety introduces atomic base types; coerce provides explicit typecasts.

\subsection{Evaluation and normalization features}

Any system built from the simple features must contain either a normal-order or a call-by-value reduction relation on terms. We prohibit inclusion of both evaluation and reduction in the same system by introducing features normalize and eval (shown in Figure 3) and defining a feature constraint normalize $\oplus$ eval (where $\oplus$ is exclusive or). For systems with computational effects, on the other hand, call-byvalue reduction must be selected. The feature eff controls inclusion of the necessary infrastructure to implement effects, and ref is built on top of it to support reference cells. 


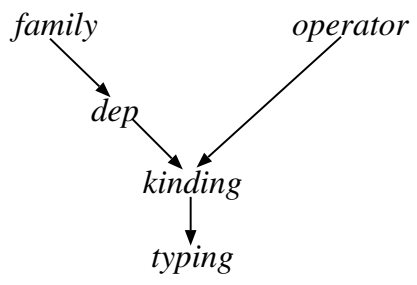

Fig. 4. Kinding features.

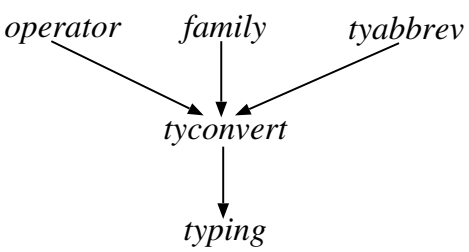

Fig. 5. Type conversion features.

\subsection{Kinding features}

systems with non-trivial well-formedness conditions on types (for example, systems with type operators or dependent type families) introduce a kinding judgement. The feature hierarchy shown in figure 4 introduces kinding as a base feature for kinding support; dep signals mutual dependency between the typing and kinding relations and introduces dependent functions; operator and family add type operators and type families respectively.

\subsection{Type conversion features}

Syntactically distinct types must be checked for "convertibility" in systems with either type abbreviations (tyabbrev) or beta-reduction on types (operator or family). The feature tyconvert (shown in figure 5) signals the presence of any one of the above three features and triggers the use of conversion testing at many points in the typing rules instead of simple type equality.

We have now defined all the necessary features for building the systems of Barendregt's lambda cube:

$\begin{array}{ll}\text { [arrow }] & \lambda \rightarrow \\ {[\text { arrow, all }]} & \text { System F } \\ {[\text { arrow, family }]} & \text { types dependent on terms } \\ \text { [arrow, all, operator }] & \text { System F }{ }^{\omega} \\ {[\text { arrow, all, operator, family }]} & \text { calculus of constructions }\end{array}$

The features all, operator, and family define the three dimensions of the cube, while arrow marks the origin point. 


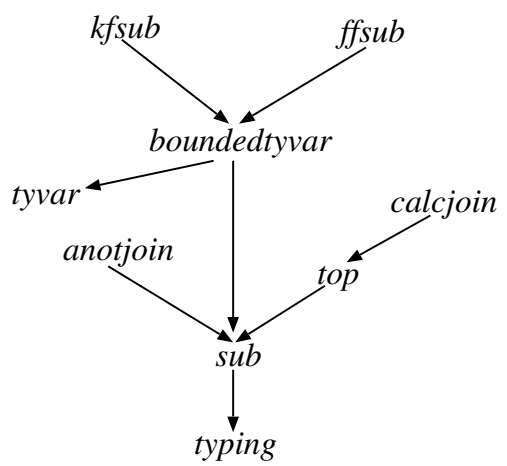

Fig. 6. Subtyping features

\subsection{Subtyping features}

A difficulty in the design of algorithmic subtyping systems arises from the nonexistence of joins in the full variant of System $F_{<}$. We want to employ the algorithm for calculating joins in systems where it makes sense, but systems that are not closed under joins must provide some other mechanism of obtaining a common supertype (for example, they might annotate "multi-armed" expressions like if and case with their intended result types). We introduce two mutually exclusive features, calcjoin and anotjoin, one of which must be specified for any system that contains a multiarmed expression. While calcjoin signals that a system contains the algorithm for calculating joins, anotjoin represents systems where multi-armed expressions must use annotated forms.

We also introduce a mutually exclusive pair of features $k f s u b$ and $f f s u b$, which characterize the "kernel" and "full" versions of $\mathrm{F}_{<:}$(Pierce, 1994). Systems including ffsub will not be closed under joins and thus cannot take advantage of the algorithm for calculating joins.

The above intuitions are formalized by the following feature constraints:

$$
\begin{aligned}
& \text { sub } \wedge(\text { bool } \vee \text { variant }) \Rightarrow \text { calcjoin } \oplus \text { anotjoin } \\
& \neg(k f \text { sub } \wedge f f \text { sub }) \\
& \neg(f f s u b \wedge \text { calcjoin })
\end{aligned}
$$

Figure 6 presents the subtyping hierarchy (boundedtyvar represents bounded type variable declarations, which are required by both variants of $\mathrm{F}_{<:}$). We can build the following familiar subtyping systems from the presented features:

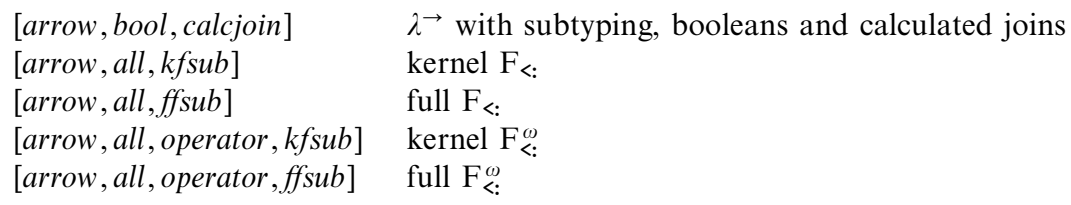




\section{Modular metatheory}

We now describe our second experiment with modularization of typed lambdacalculi. Here, our focus is not on implementations, but on modularized proofs of basic metatheoretic properties such as subject reduction and progress theorems.

In modularizing proofs, we followed exactly the same lines as the modular presentations of the systems themselves. Just as systems are cut up into clauses annotated with features, so proofs are cut up into individual cases and annotated with these same features. Just as we assemble the various judgements of a system by collecting the appropriate inference rules and printing them out with headers like "the typing relation is the least relation closed under the following rules," we likewise assemble proofs by collecting the appropriate clauses and printing them with headers like "by induction on typing derivations."

Of course, just as it is possible to generate nonsensical type systems because of errors in the repository, the "proofs" that are assembled in this way are not guaranteed to be well formed; strictly speaking, they are proof scripts, whose validity must be checked after the fact (we consider some alternatives to this approach in section 8). In our present repository, proofs are presented in standard mathematical English, and their validity must be checked by hand. Ultimately, one would like to present them in the form of proof scripts for some automated proof checker. We see no reason, in principle, why this would not be possible, but clearly much depends on the proof checker involved.

One particular caveat is that there are some properties, such as inversion lemmas (e.g. "if the statement $\Gamma \vdash t_{1} t_{2}: T$ is derivable, then, for some $S$, the statements $\Gamma \vdash \mathrm{t}_{1}: \mathrm{S} \rightarrow \mathrm{T}$ and $\Gamma \vdash \mathrm{t}_{2}: \mathrm{S}$ are derivable"), whose proofs, strictly speaking, require global reasoning. In an informal proof, this global reasoning is often camouflaged: only the case for the type constructor in question ( $\rightarrow$ in this case) is interesting, and the rest can be dealt with by a single offhand remark like "none of the other inference rules could apply here, because their conclusions are inconsistent with the shape of the term that we are considering." As proofs become more formal, however, it may become necessary to argue, for each of the other rules, that it cannot occur here (i.e. to prove a statement specifically about the arrow elimination form), we may need to perform a complete case analysis involving all the other type constructors. How much of a problem this is in practice will doubtless depend on the sophistication of the proof checker being used to verify the script assembled by TinkerType: if the checker can discover some simple proofs on its own, then even proofs of properties like inversion may appear local. (In the worst case, we may be faced with a proliferation of $n^{2}$ cases that need to be written out explicitly for proofs of properties like inversion $-n$ being the total number of features corresponding to type constructors. This would certainly be annoying, but not necessarily debilitating if the number of such global arguments is not too large.)

The repository described in this section covers a smaller collection of features than the one described in section 5. It includes numbers, booleans, functions, subtyping, polymorphism, and mutable references, plus one significant extension to the systems that we have seen up to now: equi-recursive types. It can be used to generate 
proofs of subject reduction and progress theorems, plus all required lemmas, for any sensible combination of these features. We begin by discussing systems without recursive types, then address recursive types in section 6.3 .

\subsection{Judgement forms}

The systems encoded in this repository may include four judgements: evaluation, typing, subtyping and type exposure.

The evaluation judgement appears in all systems. In its simplest form it relates terms to terms: $t \longrightarrow t$. In systems with effects (identified by the feature store), the evaluation relation is defined in store-passing style and has the form: $t ; \mu \longrightarrow t ; \mu$.

The typing judgement is also present in every system. In its simplest form, it relates a term to a type: $t$ : T. In systems with term variables (feature tmvar) the typing judgement also carries a context: $\Gamma \vdash t: \mathrm{T}$. Orthogonally, in systems with mutable references (feature store), the typing relation includes a store typing: $\Sigma \vdash t: \mathrm{T}$. In systems with both tmvar and store, the typing judgement has the form $\Gamma ; \Sigma \vdash \mathrm{t}: \mathrm{T}$.

The subtyping judgement appears only in systems including the feature $s u b$. In simple subtyping systems, the subtyping judgement relates pairs of types: $\mathrm{T}<: \mathrm{T}$. In systems with bounded quantification (feature $f s u b$ ), we must also track bounds of type variables by extending contexts with subtyping assumptions and annotating the subtyping relation with a context: $\Gamma \vdash \mathrm{T}<\mathrm{T}$.

Systems with bounded quantification also require a type exposure judgement, whose goal is to reveal the least concrete supertype of a type variable: $\Gamma \vdash \mathrm{T} \Uparrow \mathrm{T}$. Unlike the other judgements, this one is not mentioned in the inference rules defining the other judgements. Rather, it is necessary for the statements of the subtyping inversion lemmas discussed below.

\subsection{Proofs}

Figure 7 shows the dependencies between the two main theorems we have encoded - type preservation and progress - and their associated lemmas. An edge from one node (lemma) to another means that the proof of the former invokes the statement of the latter. The lemmas fall into several groups: various forms of substitution (SUBS1-SUBS4), covering substitution of both types and terms into typing and subtyping judgements, permutation (PERM1-PERM4), weakening (TWEAK and S-WEAK), inversion properties of the typing judgement (TABS-INV through ABS-INV) and subtyping judgement (ALL-INV through NAT-INV), and canonical forms properties (TABS-CANON through NAT-CANON). COMM-SUBS states a commutation property of substitutions; E-EXP is preservation of typing in systems with references under extensions of the store.

Each node is tagged with the features characterizing the systems in which it is relevant. For instance, the system of arithmetic expressions built from features nat and bool needs the successor inversion lemma (SUCC-INV), and the canonical forms lemmas for booleans and numeric values (BOOL-CANON and NAT-CANON) in addition to the two main theorems. 


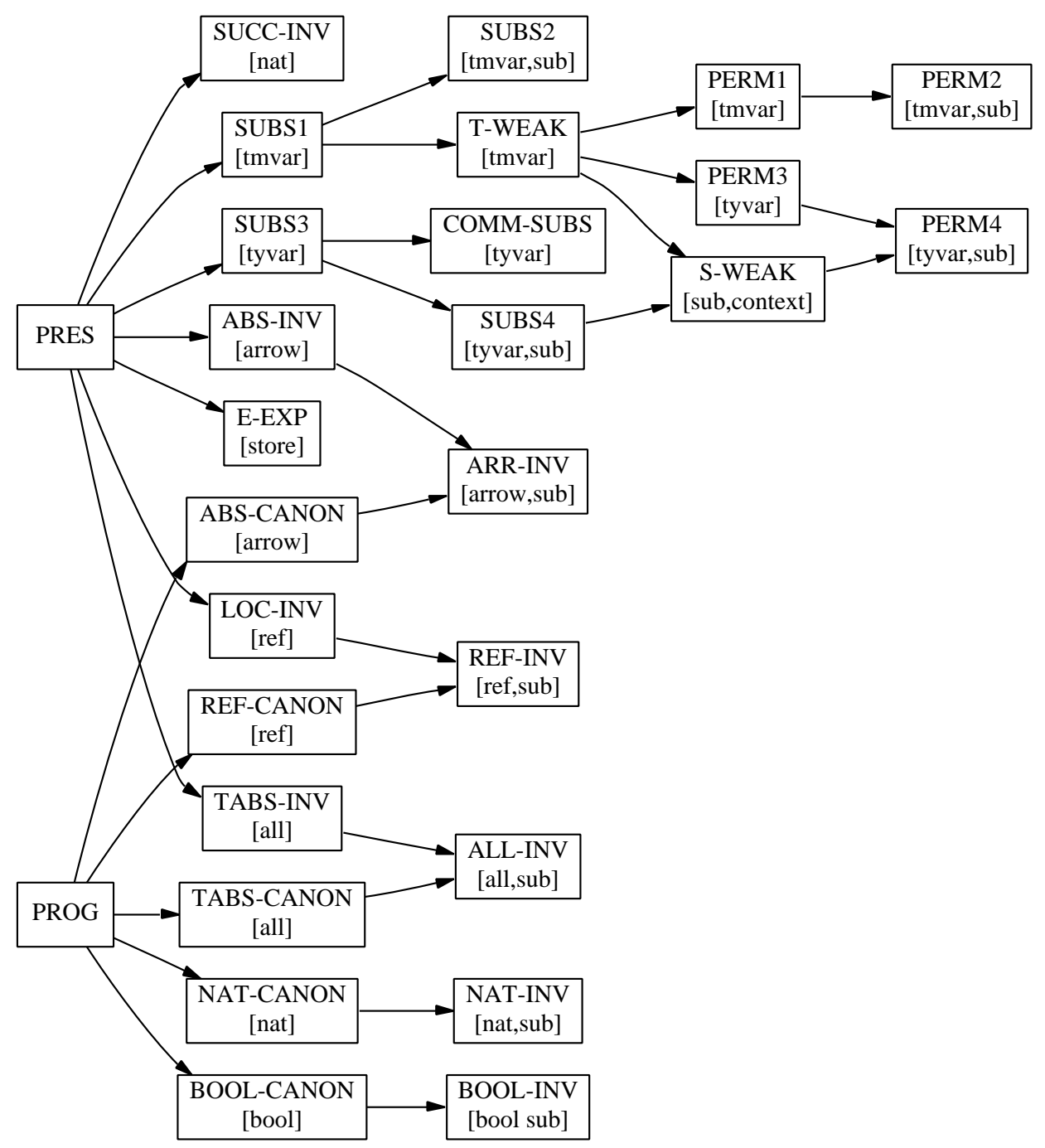

Fig. 7. Preservation and progress theorems and their lemmas.

\subsection{Recursive types}

The proofs we have described can all be encoded in TinkerType quite compactly. (Roughly, the overhead introduced by modularization is about half again as long as an ordinary, monolithic proof for a system combining all possible features.) In essence, this is because systems involved are fundamentally fairly similar.

The introduction of equi-recursive types, on the other hand, has more serious consequences. First, we must confront the issue of type equivalence. Whenever in non-recursive systems we indicated the syntactic equivalence of two types by using the same metavariable for both of them, in systems with recursive types, we must instead allow the two types to be syntactically distinct but equivalent "modulo 
unfolding" of recursive types. One example of a clause affected by the new notion of equivalence is the universal type subtyping rule in system kernel $F_{<:}$.

$$
\begin{array}{cr}
\frac{\Gamma, \mathrm{X}<: \mathrm{T} \vdash \mathrm{T}_{1}<: \mathrm{T}_{2}}{\Gamma \vdash \forall \mathrm{X}<\mathrm{T} . \mathrm{T}_{1}<: \forall \mathrm{X}<\mathrm{T} . \mathrm{T}_{2}} & \text { S-ALL }[k f s u b] \\
\frac{\Gamma, \mathrm{X}<: \mathrm{T} \vdash \mathrm{T}_{1}<: \mathrm{T}_{2} \quad \mathrm{~T} \equiv \mathrm{T}^{\prime}}{\Gamma \vdash \forall \mathrm{X}<\mathrm{T} . \mathrm{T}_{1}<: \forall \mathrm{X}<\mathrm{T}^{\prime} . \mathrm{T}_{2}} & \text { S-ALL }[k f \text { sub }, r e f]
\end{array}
$$

The equivalence relation is defined coinductively as the greatest fixed point of the equivalence rules. Similarly, we define the subtyping relation coinductively. This allows us to inherit most of the subtyping rules from the earlier systems while changing the interpretation of the rules from inductive to coinductive.

To encode these mechanisms, we create a number of new features. Feature rec identifies systems with equi-recursive types. The equivalence relation, identified by $e q$, is needed in the kernel $\mathrm{F}_{<:}$system to define the universal type subtyping rule. Similarly, we need it in systems with reference cells to define the invariant subtyping rule for type Ref $\mathrm{T}$. In the full $\mathrm{F}_{<}$system, on the other hand, the equivalence relation is unnecessary, because it is subsumed by the subtyping relation. These relations are encoded in the feature constraint $r e c \Rightarrow(f f s u b \wedge \neg r e f) \vee e q$.

The features indsub and coindsub offer a choice between two different versions of subtyping relations. In the presence of recursive types, we must use coinductive subtyping - this is captured by the constraint rec $\wedge s u b \Rightarrow$ coindsub. Also, any subtyping system must choose between the the inductive or coinductive view: $s u b \Rightarrow$ indsub $\vee$ coindsub. Finally, these two views are mutually exclusive: $\neg($ indsub $\wedge$ coindsub).

The inclusion of recursive types results in a significant increase in the size of the repository. First, the introduction of the equivalence judgement necessitates creation of new equivalence rules and various new properties and their proofs. Similarly, new properties are required for the new subtyping relation. Transitivity is one of them: while in inductive subtyping systems, we can explicitly stipulate that subtyping is transitive by including the S-TRANS rule, we may not do so in a coinductive system. (This rule renders a coinductively defined subtyping relation total (Gapeyev et al., 2000).)

More severely, the new repository must maintain two parallel, but totally unrelated, hierarchies of proof cases. Any theorem or lemma that used to be proved by induction on subtyping derivations, must basically be re-proved from scratch in the coinductive framework. (Fortunately, the properties of the typing relation remain largely unchanged by the addition of recursive types.)

\section{Related work}

The initial inspiration for our work came from the type system fragments used by Abadi and Cardelli in their book, A Theory of Objects (1996). There, the repository consists of a collection of named "fragments" analogous to our components. A system is specified by naming a collection of fragments whose contents are 
to be concatenated. This is a degenerate instance of our framework, where each component is labeled with a single, distinct feature and where there is no dependency between features, and no static consistency checks are performed. Their book presents a substantial collection of fragments, covering (declarative formulations of) approximately the same range of type systems as the ones described here in section 5 .

Another close relative of our work is Prehofer's feature-oriented programming (1997). Like our approach, it includes features and dependencies between them, components with multiple variants, and an assembly process that combines appropriate variants based on a set of requested features. The main difference is the application domain. Our approach focuses on formal systems, and the basic unit of composition is an individual inference rule. Feature-oriented programming is used to assemble objects; its basic unit of composition is a group of related methods. Prehofer introduces an extension of Java with feature support and describes two approaches for compiling it into Java.

The Hyperspace project (Ossher \& Tarr, 1999; Tarr et al., 1999) proposes a general theory of multi-dimensional separation of concerns. In this work, units are atomic entities similar to our clauses. A unit can be related to several concerns, which correspond to our features. Concerns are partitioned into orthogonal dimensions. Hyperslices are composed of units and resemble our components. They can be merged to form hypermodules that are similar to systems in our work. This approach is somewhat more abstract than ours - for example, the algorithm for merging hyperslices is taken as a parameter.

Earlier work in the same group promoted a technology called subject-oriented programming (Harrison \& Ossher, 1993). One of its principal goals was to allow parallel development of classes and provide a composition mechanism to obtain a final system. In this view, classes resemble our components, and their merging is analogous to system assembly. No mechanism corresponding to features is provided.

Aspect-oriented programming (Kiczales et al., 1997; Kiczales, 1996) starts from the observation that it is sometimes difficult to address certain issues in a program without obscuring its main functionality. These issues, called aspects, "crosscut" the natural decomposition of the main functionality, resulting in small bits of related code strewn across the system. To simplify designing programs with these properties, AOP proposes using conventional component languages to implement basic functionality, and special purpose aspect languages to deal with the cross-cutting issues. A special process called weaving merges programs written in these languages to produce the resulting system. To some extent, we can view our language of features, clauses, and components as a particular aspect language; the component language is whatever language is used to express the contents of clauses.

Another area of related work is monadic techniques for structuring interpreters and compilers (Steele, 1994; Liang et al., 1995; Espinosa, 1995; Liang \& Hudak, 1996; Harrison \& Kamin, 1998). The focus here is on modular definition and combination of different aspects of computation (state, exceptions, concurrency, etc.). It is a highly structured approach, using the type system of the metalanguage to 
control the composition process and focusing on constraints arising from interaction between features. It does not appear easy to extend the monadic approach to typing features in the spirit of the present work. On the other hand, we believe that a monadic style could be used to structure the presentation of the operational semantics of our typed lambda-calculi.

\section{Conclusions and future work}

The TinkerType formalism, its implementation, and our repository of typed lambdacalculi have evolved in parallel over many months. At present, our larger repository contains about 14,000 lines of TinkerType sources, of which roughly $20 \%$ is TeX sources in the bodies of clauses, $60 \%$ is ML clauses, and $20 \%$ is TinkerType proper. From this, we routinely generate about 80 different typecheckers, totaling over 120,000 lines of ML. Maintaining all these checkers by hand would be next to impossible.

Our focus in this paper has been on using TinkerType to define typed lambdacalculi. We believe that TinkerType at its present stage is most useful in this area which includes developing and studying families of programs or mathematical definitions that are naturally structured as collections of rules. In addition to lambdacalculi this includes other programming calculi (e.g. Abadi and Cardelli's Object Calculi, process calculi, etc.), compilers, and a wide variety of logics, as well as programs from other domains such as expert systems. TinkerType is especially useful for systems with a large number of interacting features that are hard to manipulate by existing logical or programming frameworks. We conjecture that TinkerType may even be helpful in developing a single program or formal system, rather than a family, since it encourages identifying the underlying features and the relationships between them thus leading to a better overall understanding of the system. More speculatively, we wonder whether ideas from TinkerType (or indeed the system itself) could be applied in the domain of software configuration management, for generating complex Makefiles or as a more principled alternative to the tangles of \#ifdef directives found in many $\mathrm{C}$ programs that are engineered for portability.

Even when we are interested in building just a single system, rather than a whole family of similar systems, there may be benefits to using a tool like TinkerType to flexibly factor the system's description according to some natural set of features. In this respect, TinkerType can be viewed as a sort of aspect-oriented programming language - or perhaps more fairly, as an aspect-oriented macro preprocessor. However, the benefits of this ability to factor and combine code fragments must be weighed against the overhead involved in cutting up the program into small pieces, tagging the pieces with features, etc.

Type systems are often formalized using proof checkers based on logical frameworks, and it is natural to wonder whether our ideas could be combined with such systems. We can imagine a variety of ways in which this idea might be approached. One would be to extend a proof-assistant with concepts drawn from TinkerType for example, we could work to enhance the assistant's theory definition language 
with TinkerType-like feature support. Rather than viewing TinkerType and the proof assistant as two separate stages (the latter checking what the former generates), the two activities would be tightly integrated. Another approach would be to add to TinkerType some of the mechanisms found in logical frameworks, such as uniform treatment of variable binding constructs. This proposal amounts to replacing the single type of clause contents that TinkerType currently manipulates (i.e. uninterpreted strings) with more specialized structures that can be understood to some extent by the tool. Recent work on a general treatments of abstract syntax and variable binding - for example, by Fiore, Plotkin and Turi (1999) - offers an important first step in this direction. Burstall and Goguen's (1984) older notion of institutions may also provide useful insight.

\section{Acknowledgments}

This work was supported by the University of Pennsylvania and by NSF grants CCR9701826, Principled Foundations for Programming with Objects and CCR-9912352, Modular Type Systems.

\section{References}

Abadi, M. and Cardelli, L. (1996) A Theory of Objects. Springer-Verlag.

Barendregt, H. P. (1992) Lambda calculi with types. In: Abramsky, S., Gabbay, Dov M. and Maibaum, T. S. E., editors, Handbook of Logic in Computer Science, vol. II. Oxford University Press.

Basin, D., Matthews, S. and Viganò, L. (1995) A modular presentation of modal logics in a logical framework. Proceedings of the Tbilisi Symposium on Language, Logic and Computation.

Berardi, S. (1988) Towards a mathematical analysis of the Coquand-Huet calculus of constructions and the other systems in Barendregt's cube. Technical report, Department of Computer Science, CMU, and Dipartimento Matematica, Universita di Torino.

Espinosa, D. (1995) Semantic Lego. PhD thesis, Columbia University.

Fiore, M., Plotkin, G. and Turi, D. (1999) Abstract syntax and variable binding (extended abstract). IEEE Symposium on Logic in Computer Science (LICS), pp. 193-202. IEEE Press.

Gapeyev, V., Levin, M. and Pierce, B. (2000) Recursive subtyping revealed. International Conference on Functional Programming (ICFP), Montreal, Canada.

Ghelli, G. (1990) Proof theoretic studies about a minimal type system integrating inclusion and parametric polymorphism. PhD thesis, Università di Pisa. (Technical report TD-6/90, Dipartimento di Informatica, Università di Pisa.)

Goguen, J. A. and Burstall, R. M. (1984) Introducing institutions. In: Clarke, E. and Kozen, D., editors, Logics of Programs: Workshop: Lecture Notes in Computer Science 164, pp. 221-256. Springer-Verlag.

Gunter, C. A. (1992) Semantics of Programming Languages: Structures and Techniques. MIT Press.

Harper, R., Honsell, F. and Plotkin, G. (1992) A framework for defining logics. J. ACM, 40(1), 143-184.

Harrison, W. and Ossher, H. (1993) Subject-oriented programming (a critique of pure objects). In: Paepcke, A., editor, ACM Symposium on Object Oriented Programming: Systems, 
Languages, and Applications (OOPSLA), pp. 411-428. (ACM SIGPLAN Notices, 28(10).) ACM Press.

Harrison, W. L. and Kamin, S. N. (1998) Modular compilers based on monad transformers. Proceedings IEEE International Conference on Computer Languages.

Jacobs, B. (1999) Categorical Logic and Type Theory. Studies in Logic and the Foundations of Mathematics, no. 141. North Holland, Elsevier.

Kiczales, G. (1996) Aspect-oriented programming. ACM Comput. Surv. 28(4), 154.

Kiczales, G., Lamping, J., Mendhekar, A., Maeda, C., Lopes, C., Loingtier, J.-M. and Irwin, J. (1997) Aspect-oriented programming. European Conference on Object-Oriented Programming (ECOOP): Lecture Notes in Computer Science 1241. Springer-Verlag.

Landin, P. J. (1965) A correspondence between ALGOL 60 and Church's lambda-notation: Parts I and II. Comm. ACM, 8(2,3), 89-101, 158-165.

Landin, P. J. (1966) The next 700 programming languages. Comm. ACM, 9(3), 157-166.

Liang, S. and Hudak, P. (1996) Modular denotational semantics for compiler construction. Programming Languages and Systems (ESOP '96), Proc. 6th European Symposium on Programming: Lecture Notes in Computer Science 1058, pp. 219-234. Springer-Verlag.

Liang, S., Hudak, P. and Jones, M. (1995) Monad transformers and modular interpreters. ACM Symposium on Principles of Programming Languages (POPL), pp. 333-343. San Francisco, CA.

Mitchell, J. C. (1996) Foundations for Programming Languages. MIT Press.

Moggi, E. (1989) Computational lambda-calculus and monads. Fourth Annual Symposium on Logic in Computer Science, pp. 14-23. (Asilomar, CA). IEEE Press.

Mosses, P. D. (1992) Action semantics. Cambridge Tracts in Theoretical Computer Science, no. 26. Cambridge University Press.

Odersky, M., Sulzmann, M. and Wehr, M. (1999) Type inference with constrained types. Theory \& Practice of Object Systems, 5(1), 35-55.

Ossher, H. and Tarr, P. (1999) Multi-dimensional separation of concerns in hyperspace. Technical Report RC 21452(96717)16APR99, IBM T. J. Watson Research Center.

Pfenning, F. and Elliott, C. (1988)) Higher-order abstract syntax. ACM SIGPLAN Conference on Programming Language Design and Implementation (PLDI), pp. 199-208. Atlanta, GA. (Available as Ergo Report 88-036, School of Computer Science, Carnegie Mellon University, Pittsburgh.)

Pierce, B. C. (1994) Bounded quantification is undecidable. Information \& Computation, 112(1), 131-165. (Also in Gunter, C. A. and Mitchell, J. C., editors, Theoretical Aspects of Object-Oriented Programming: Types, Semantics, and Language Design, MIT Press, 1994.)

Prehofer, C. (1997) Feature-oriented programming: A fresh look at objects. In: Aksit, M. and Matsuoka, S., editors, European Conference on Object-Oriented Programming (ECOOP): Lecture Notes in Computer Science 1241. Springer-Verlag.

Steele, Jr., G. L. (1994) Building interpreters by composing monads. ACM Symposium on Principles of Programming Languages (POPL), pp. 472-492. Portland, OR. ACM Press.

Tarr, P., Ossher, H. L., Harrison, W. H. and Sutton, Jr., S. M. (1999) N degrees of separation: Multi-dimentional separation of concerns. Proceedings of the 21st International Conference on Software Engineering.

Terlouw, J. (1989) Een nadere bewijstheoretische analyse van GSTTs. Manuscript, University of Nijmegen, Netherlands. 